\title{
Aberrant C-terminal domain of polymerase $\eta$ targets the functional enzyme to the proteosomal degradation pathway
}

\author{
Sana Ahmed-Seghir ${ }^{a}$, Caroline Pouvelle ${ }^{a}$, Emmanuelle Despras $^{a}$, Agnès Cordonnier $^{\mathrm{b}}$, \\ Alain Sarasin ${ }^{a}$, Patricia L. Kannouche ${ }^{\mathrm{a}, *}$ \\ a Université Paris-Sud, CNRS-UMR8200, Equipe labellisée Ligue Contre le Cancer, Gustave Roussy, Villejuif, France \\ b CNRS-FRE3211, IREBS, BP 10413, F-67412 Illkirch-Cedex, France
}

\section{A R T I C L E I N F O}

\section{Article history:}

Received 11 November 2014

Received in revised form 14 February 2015

Accepted 16 February 2015

Available online 26 February 2015

\section{Keywords:}

Translesion synthesis

$\mathrm{XP}-\mathrm{V}$

Xeroderma pigmentosum variant

Bortezomib

MG132

Ubiquitin/proteasome system

Ultraviolet

\begin{abstract}
A B S T R A C T
Xeroderma pigmentosum variant (XP-V) is a rare genetic disease, characterized by sunlight sensitivity and predisposition to cutaneous malignancies. XP-V is caused by a deficiency in DNA polymerase eta (Pol $\eta$ ) that plays a pivotal role in translesion synthesis by bypassing UV-induced pyrimidine dimers. Previously we identified a new Pol $\eta$ variant containing two missense mutations, one mutation within the bipartite NLS (T692A) and a second mutation on the stop codon (X714W) leading to a longer protein with an extra 8 amino acids (721 instead of 713 AA). First biochemical analysis revealed that this Pol $\eta$ missense variant was barely detectable by western blot. As this mutant is extremely unstable and is nearly undetectable, a definitive measure of its functional deficit in cells has not been explored. Here we report the molecular and cellular characterization of this missense variant. In cell free extracts, the extra 8 amino acids in the C-terminal of Pol $\eta^{721}$ only slightly reduce the bypass efficiency through CPD lesions. In vivo, Pol $\eta^{721}$ accumulates in replication factories and interacts with mUb-PCNA albeit at lower level than Pol $\eta^{\text {wt }}$. XP-V cells overexpressing Pol $\eta^{721}$ were only slightly UV-sensitive. Altogether, our data strongly suggest that Pol $\eta^{721}$ is functional and that the patient displays a XP-V phenotype because the mutant protein is excessively unstable. We then investigated the molecular mechanisms involved in this excessive proteolysis. We showed that Pol $\eta^{721}$ is degraded by the proteasome in an ubiquitin-dependent manner and that this proteolysis is independent of the E3 ligases, CRL4 ${ }^{\text {cdt2 }}$ and Pirh2, reported to promote Pol $\eta$ degradation. We then demonstrated that the extra 8 amino acids of Pol $\eta^{721}$ do not act as a degron but rather induce a conformational change of the Poln C-terminus exposing its bipartite NLS as well as a sequence close to its UBZ to the ubiquitin/proteasome system. Interestingly we showed that the clinically approved proteasome inhibitor, Bortezomib restores the levels of Pol $\eta^{721}$ suggesting that this might be a therapeutic approach to preventing tumor development in certain XP-V patients harboring missense mutations.
\end{abstract}

(c) 2015 Elsevier B.V. All rights reserved.

\section{Introduction}

Most types of DNA damage block replication fork progression during DNA synthesis because replicative DNA polymerases are unable to accommodate altered DNA bases in their active sites. To overcome such blocks, eukaryotic cells employ a specialized set of DNA polymerases that carry out translesion synthesis (TLS) past DNA lesions [8]. These polymerases, mostly belonging to the Y family, have a more open conformation and can accommodate different damaged bases in their active sites [37]. While these catalytic sites are conserved between different family members and

\footnotetext{
* Corresponding author. Tel.: +331421140 30; fax: +331421150 08.

E-mail address: patricia.kannouche@gustaveroussy.fr (P.L. Kannouche).
}

are typically contained within the N-terminal 432 amino acids, the 250 C-terminal amino acids are poorly conserved. This C-terminal region is mainly involved in the cellular localization and regulation of these TLS polymerases [18,19,25,34]. The best characterized of these Y-family polymerases is DNA polymerase (Poln), which participates in genome stability through its capacity to accurately bypass the most abundant photoproduct, the TT-CPD $[13,24]$. Mutations in the human POLH gene encoding Pol $\eta$ are responsible for the cancer-prone xeroderma pigmentosum variant (XP-V), a human recessive disorder characterized by a high sensitivity to solar light and a dramatic predisposition to cutaneous tumors. XP-V cells present a replication defect and an elevated mutagenesis upon UV exposure [23,32].

Because Poln is error-prone on undamaged DNA, it has to be tightly regulated in cells (for review [28]). A critical step in the 
regulation of TLS is the post-translational modification of proliferating cell nuclear antigen (PCNA), the replicative sliding clamp that plays an essential role in DNA replication [11]. Upon UV irradiation, PCNA is monoubiquitinated on its Lys-164. This event is considered to facilitate the TLS process since Pol $\eta$ preferentially interacts with monoubiquitinated PCNA through its ubiquitin-binding domain (UBZ) as well as its PCNA interacting peptide (PIP)-box [2,20,36].

Recent works showed that the activity of Poln can also be regulated by its monoubiquitination [3]. It was hypothesized that, Pol $\eta$ is mono-ubiquitinated on one of the four C-terminal lysines at the end of the protein to inhibit its interaction with PCNA in undamaged cells [3]. Other studies highlight different mechanisms to promote the degradation of Poln. In Caenorhabditis elegans (C. elegans), the GEI-17 SUMO E3 ligase protects PolH protein from degradation mediated by CRL4 ${ }^{\text {Cdt2 }}$ pathway [21]. It has been reported that in human cells, the RING-H2 type E3 ligase (Pirh2) promotes Poln degradation in an ubiquitin-independent manner [16]. In addition, the Murine double minute-2 (MDM2) can also serve as an E3 ligase for Pol $\eta$ polyubiquitination and proteosomal degradation in response to ultraviolet irradiation [17].

The vast majority of XP-V patients harbor $P O L H$ mutations giving rise to a truncated protein. However, some XP-V patients have missense mutations producing full-length Pol $\eta$ proteins that are generally undetectable by western blot [26]. We recently identified a new Poln variant containing two missense mutations, one mutation within the bipartite NLS (T692A) and a second mutation on the physiological stop codon (X714W) leading to a longer protein with an extra 8 amino acids (721 instead of 713 AA [26]). As the Pol $\eta$ missense variant is extremely unstable and is nearly undetectable, a definitive measure of its functional deficit in cells has not been explored.

In the current work, we report the characterization of the Poln variant, Pol $\eta^{721}$ at the molecular and cellular level. We found that Pol $\eta^{721}$ is functional in vitro and in vivo and that the patient displays a XP-V phenotype because the mutant protein is excessively unstable. We also demonstrated that the extra 8 amino acids of Pol $\eta^{721}$ do not act as a degron but rather induce a conformational change of the Pol $\eta \mathrm{C}$-terminus exposing its bipartite NLS as well as a sequence close to its UBZ to the ubiquitin/proteasome system. Interestingly we showed that a clinically approved proteasome inhibitor, Bortezomib fully restores the levels of Pol $\eta^{721}$ suggesting that this might be a therapeutic approach to preventing tumor development in certain XP-V patients harboring missense mutations.

\section{Materials and methods}

\subsection{Cell culture and treatments}

Normal and XP-V primary diploid fibroblasts (405VI and XP872VI respectively), SV-40 immortalized fibroblasts XP-V (named XP30RO) and SV-40 immortalized human fibroblasts MRC5 were cultivated in Minimal Eagle Medium (MEM; Gibco) supplemented with $10 \%$ fetal calf serum (FCS), L-glutamine, $100 \mathrm{U} / \mathrm{ml}$ penicillin and $100 \mu \mathrm{g} / \mathrm{ml}$ streptomycin under $5 \% \mathrm{CO}_{2}$. HEK293FT cells (Invitrogen) were cultivated in D-MEM (Dulbecco's Modified Eagle medium) containing sodium pyruvate, penicillin/streptomycin and $10 \%$ FCS under $5 \% \mathrm{CO}_{2}$. Cycloheximide and MG132 purchased from Sigma were used at $25 \mu \mathrm{g} / \mathrm{ml}$ and $10 \mu \mathrm{M}$ respectively. Bortezomib was purchased from Calbiochem and used as indicated. Global and local UV-C irradiations were performed as previously described [18].

\subsection{Constructions and transfections}

All constructs for mutant analysis were generated using standard molecular biology techniques and were verified directly by DNA sequencing. The parent plasmid for expression of Pol $\eta^{721}$ and its various mutant derivatives was pCDNA-POLH [32], which contains a full-length $P O L H$ cDNA under the control of the CMV promoter. Point mutants were generated by PCR-based methods using a QuikChange ${ }^{\circledR}$ site-directed mutagenesis kit (Stratagene) according to the manufacturer's instructions. Details on all constructs are available upon request.

Transient transfection was performed using Exgen500 (Fermentas) according to the manufacturer's protocol, and cells were analyzed $24-48 \mathrm{~h}$ later. For stable transfection, XP30RO fibroblasts were transfected using FuGENE 6 (Roche) and stable Pol $\eta^{721}$ transfectants were isolated and further propagated in medium containing $100 \mu \mathrm{g} / \mathrm{ml}$ zeocin (InvivoGen). Stable XP30RO cell lines expressing wild-type Pol $\eta$ (Pol $\eta^{\mathrm{wt}}$ ) or Pol $\eta^{\mathrm{D} 652 \mathrm{~A}}$ have been previously described ([7,32], respectively).

siRNA were purchased from Eurogentec: siRNA PCNA [GCCGAGAUCUCAGCCAUAU], and Dharmacon: non-targeting siRNA [ON-TARGETplus non target pool], siRNA Cdt2 [ON-TARGETplus SMART pool] and siRNA Pirh2 [CCAACAGACUUGUGAAGAA]. Cells were transfected with $10 \mathrm{nM}$ of siRNAs using INTERFERin reagent (Polyplus) according to the manufacturer's instruction, and analyzed $72 \mathrm{~h}$ later.

\subsection{Antibodies}

Antibodies used for western blotting and immunofluorescence were purchased from Abcam (Poln ab17725, Cdt2, GAPDH, GFP), Santa Cruz (Chk1, Pol H-300, Lamin A/C, PCNA, Nbs1,), Bethyl (pS33RPA 32, Cdt1, Pol $\eta)$, Sigma ( $\beta$-actin), BD biosciences ( $\beta$-catenin), Calbiochem (RPA32), Cell signaling (Ps345-Chk1), and Novus biological (Ps343-NBS1).

\subsection{Cell lysates and western blot}

For analysis of phosphorylated proteins, cells were scrapped directly in NETN buffer $(150 \mathrm{mM} \mathrm{NaCl}, 1 \mathrm{mM}$ EDTA, $50 \mathrm{mM}$ Tris pH 7.5, 0.5\% NP-40, antiproteases, antiphosphatases (PhosSTOP, Roche)) and incubated $30 \mathrm{~min}$ on ice. Extracts were sonicated $15 \mathrm{~s}$ at amplitude $30 \%$. Whole cell extracts were prepared in lysis buffer (20 mM NaCl, $1 \mathrm{mM} \mathrm{MgCl}$, $50 \mathrm{mM}$ Tris $\mathrm{pH} 7.5,0.1 \%$ SDS, protease inhibitor cocktail and $50 \mathrm{U} / \mathrm{ml}$ benzonase, Novagen). Proteins were separated by electrophoresis in 8-10\% SDS-PAGE gels and analyzed by western-blotting.

\subsection{Immunoprecipitation, His pull-down and cell fractionation}

Immunoprecipitations of PCNA were performed as previously described [20]. For His pull-down, HEK 293FT cells were lysed in denaturing conditions ( $100 \mathrm{mM} \mathrm{NaH}_{2} \mathrm{PO}_{4}, 10 \mathrm{mM}$ Tris-Cl, $8 \mathrm{M}$ urea, $20 \mathrm{mM}$ imidazole, $\mathrm{pH} 8$ ), centrifuged and supernatant was incubated with Ni-NTA-agarose (Quiagen) during $45 \mathrm{~min}$ at RT before washes and a denaturation by heating.

To obtain chromatin-bound proteins cell fractionation was performed as already described [7]. Nuclear/cytosolic fractionation was performed using 1 volume of isotonic buffer $(50 \mathrm{mM}$ Tris- $\mathrm{HCl}$ $\mathrm{pH} 7.5,10 \mathrm{mM} \mathrm{MgCl}_{2}, 15 \mathrm{mM} \mathrm{CaCl} 2,1.5 \mathrm{mM}$ sucrose and $0.05 \%$ NP40). After incubation, extracts were centrifuged. The supernatant (cytoplasmic fraction) was then transferred in a new tube and kept at $-80^{\circ} \mathrm{C}$ and nuclei were resuspended in 3 volumes of Nuclear protein extraction buffer $\left(20 \mathrm{mM}\right.$ Hepes, $1.5 \mathrm{mM} \mathrm{MgCl}_{2}, 0.42 \mathrm{M}$ $\mathrm{NaCl}, 0.2 \mathrm{mM}$ EDTA and $25 \%$ glycerol) during $30 \mathrm{~min}$ at $4{ }^{\circ} \mathrm{C}$ then sonicated. After centrifugation, the nuclear soluble fraction was heat-denaturated. 


\subsection{Proliferation assay}

Cells were plated at $1.5 \times 10^{5}$ per well of a six-well plate $24 \mathrm{~h}$ before UVC exposure. Seventy-two hours after irradiation and incubation in medium supplemented or not with $0.38 \mathrm{mM}$ caffeine (Sigma), cells were counted with trypan blue using Neubauer haemocytometer.

\subsection{Immunofluorescence}

Cells were seeded on glass coverslips $24 \mathrm{~h}$ before UVC irradiation. For total Poln immunostaining, cells were fixed in $4 \%$ paraformaldehyde and permeabilized with $0.5 \%$ Triton-X100 in PBS. For detection of replication foci, soluble proteins were extracted in CSK100 (100 mM NaCl, $300 \mathrm{mM}$ sucrose, $3 \mathrm{mM} \mathrm{MgCl}_{2}$, $10 \mathrm{mM}$ PIPES pH 6.8, $1 \mathrm{mM}$ EGTA, 0.2\% Triton X100, antiproteases (Complete EDTA free, Roche)) for 5 min on ice under gentle agitation. Cells were washed in ice-cold PBS and fixed in $4 \%$ formaldehyde. Coverslips were immersed in methanol at $-20^{\circ} \mathrm{C}$ for $10 \mathrm{~s}$ (only for PCNA immunodetection). After washes in PBS, coverslips were blocked in IF buffer (3\% BSA, 0.5\% Tween 20 in PBS) for $30 \mathrm{~min}$. Antibodies were diluted in IF buffer. Primary antibodies (1/500 rabbit anti-pol $\eta$ Abcam ab17725, 1/500 PCNA PC10 Santa Cruz) were incubated for $1 \mathrm{~h}$ at room temperature. Secondary antibodies (1/1000 anti-rabbit Alexa Fluor 488, 1/1000 anti-mouse Alexa Fluor 594) were incubated for $30 \mathrm{~min}$ at room temperature. Coverslips were mounted in mounting medium (Dako) supplemented with DAPI (Sigma). Images were acquired on an Axio Imager Z1 microscope using the Axio Vision software (Zeiss). Immunodetection of Poln and PCNA after local UV-irradiation was performed as previously described [18].

\section{Results}

\subsection{Aberrant C-terminal region of Poln directs the protein to the degradation in the nucleus}

We previously reported the identification of a XP-V patient (called XP872VI) harboring two missense mutations at the homozygous state that are not located in the catalytic domain of the DNA polymerase: one missense mutation in the bipartite NLS (T692A) and a second missense mutation on the physiological stop codon (X714W) leading to a longer protein with another stop codon eight amino-acids downstream (721 instead of 713 AA, Fig. 1A). First biochemical analysis revealed that this mutant was barely detectable by western blot suggesting that this protein is unstable [26]. Consistent with this hypothesis we found similar levels of POLH mRNA in XP872VI and 405VI cells (data not shown).

In order to better understand the cause of this instability, we tested whether this Poln missense variant was able to be stabilized by the proteasome inhibitor MG132. Primary fibroblasts from the XP872VI patient were analyzed by western-blotting and compared to primary 405VI cells expressing normal level of Poln. As expected, no detectable band was observed in XP872VI cells whereas in wildtype cells, a band was detected at around $80 \mathrm{kDa}$ (Fig. 1B). After incubation of XP-V cells with MG132 for $3 \mathrm{~h}$ a very faint band was observed suggesting that, in XP872VI cells the Pol $\eta$ variant is excessively degraded by the proteasome.

To better understand the mechanism responsible for this proteolysis, we generated a POLH cDNA harboring the missense mutation on the stop codon leading to a longer protein with an extra 8 amino acids (that will be called Pol $\eta^{721}$ in the rest of the paper). We then stably expressed this mutant in XP30RO fibroblasts, a SV40-transformed XP-V cell line which does not express Pol $\eta$ because of a point mutation in the $P O L H$ gene causing a premature stop codon [35]. As control, we used the previously described XP30RO cell line stably expressing Poln ${ }^{\mathrm{WT}}$ [32]. We confirmed that XP30RO-Pol $\eta^{721}$ cell line recapitulates the stability of Pol $\eta$ observed in XP872VI cells with a very weak level of the protein in untreated cells and its stabilization $3 \mathrm{~h}$ after MG132 treatment (Fig. 1C). It is noteworthy that in primary XP872VI cells, inhibition of the proteasome hardly restores Poln variant levels (Fig. 1A) while we observed a nice Pol $\eta^{721}$ recovery in the SV40-transformed XP-V fibroblasts after MG132 treatment (Fig. 1C). We assume that this difference is mainly due to the fact that in primary cells $\mathrm{POLH}$ gene expression is under the control of its own promoter which is quite weak while in XP30RO-Pol $\eta^{721}$ cells, POLH cDNA expression is under the control of the CMV promoter known to be very strong. Therefore in the clone the production of Pol $\eta^{721}$ protein is very high and this can be observed as soon as the proteasome is inhibited.

The C-terminal part of Poln contains an UBZ, NLS and PIP motifs and this region is mainly involved in the cellular localization and regulation of the protein. We reasoned that the extra-C-terminal sequence ( 8 amino acids) of Pol $\eta^{721}$ could affect the cellular distribution of the protein leading to its degradation. We thus examined the distribution of Pol $\eta^{721}$ after cell fractionation. We showed that after treatment with MG132, Pol $\eta^{721}$ is mainly found in the nuclear fraction as observed for Pol $\eta^{\text {wt }}$ (Fig. 1D). This was confirmed by immunofluorescence experiments after transient transfection of plasmids expressing Pol $\eta^{\mathrm{wt}}$, Pol $\eta^{\mathrm{T} 692 \mathrm{~A}}$ or Pol $\eta^{721}$ in XP30RO cells and treatment with MG132. We clearly observed a nuclear localization for all Pol $\eta$ variants (Fig. S1). These results also indicate that the missense mutation within the NLS (T692A) does not affect Pol $\eta$ localization.

To determine the sub-nuclear localization of Pol $\eta^{721}$, we performed another type of cell fractionation. XP30RO-Pol $\eta^{721}$ cells were first treated or not with MG132 for $1 \mathrm{~h}$ then cells were lysed in total lysis buffer (WCE) or in CSK buffer to remove soluble proteins and the resulting pellet mainly contained chromatin fraction (CF) Western blotting analysis revealed that the majority of Pol $\eta^{721}$ is retrieved in chromatin fraction (Fig. 1E) suggesting that the extraC-terminal part of Pol $\eta^{721}$ does not abolish the capability of the protein to bind chromatin.

\subsection{Poln $\eta^{721}$ is a functional enzyme which can complement $X P-V$ cells when it is overexpressed}

As Pol $\eta^{721}$ is extremely unstable and is nearly undetectable, a definitive measure of its functional deficit in cells has not been explored. Indeed, we do not know whether the XP872VI patient displays a XP-V phenotype because the mutant protein is functional but excessively unstable or if it is not functional and the residual protein cannot bypass UV-induced DNA damage. Therefore we examined if Pol $\eta^{721}$ was proficient for the lesion bypass in vitro and in vivo. We first assessed the ability of Pol $\eta^{721}$ to bypass CPD lesion in vitro by using XP-V cell extracts complemented with different Pol $\eta$ proteins produced in reticulocyte lysates (Fig. S2A). We analyzed the bypass efficiencies of Pol $\eta^{\mathrm{wt}}$, Pol $\eta^{721}$ and Pol $\eta^{\text {DEAD }}$, a catalytically inactive form of Pol $\eta$ (as a negative control). We observed that the Pol $\eta^{\text {wt }}$ and Pol $\eta^{721}$ were able to bypass CPD. However, at similar protein levels, Poln $\eta^{721}$ showed a lesser efficiency for bypassing CPD lesions than Pol $\eta^{\text {wt }}$ (59\% vs 75\%) suggesting that the extra 8 amino acids of Pol $\eta^{721}$ slightly impact on the folding of the C-terminal of Pol $\eta$ required for an efficient CPD bypass in human cell free extracts [29].

Next, we analyzed the nuclear localization of Pol $\eta^{721}$ in UVirradiated cells. As shown in Fig. 2A, $1 \mathrm{~h}$ after MG132 treatment, Pol $\eta^{721}$ is able to accumulate in replication factories where it colocalizes with PCNA in UV-irradiated cells as observed for Pol $\eta^{\mathrm{wt}}$. This was confirmed after local UV-irradiation (Fig. S2B). 
A

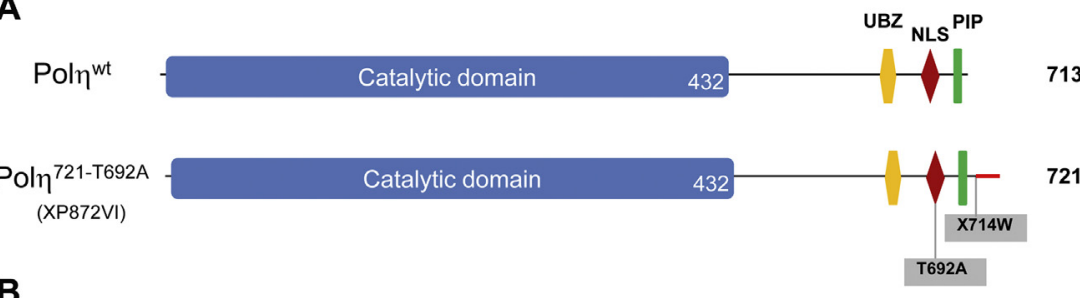

B

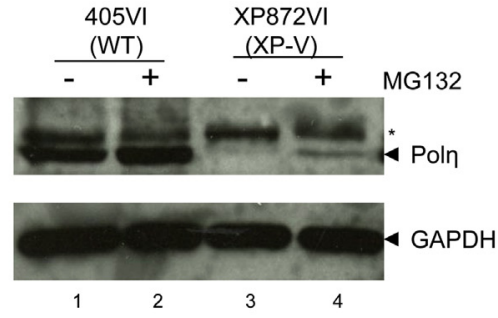

C

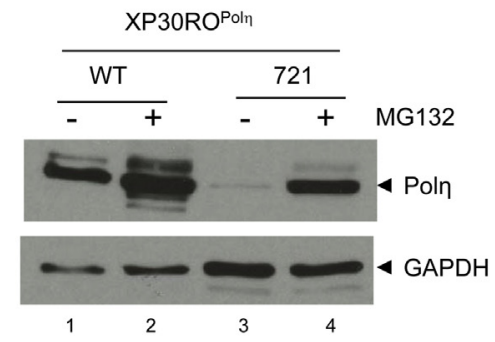

D

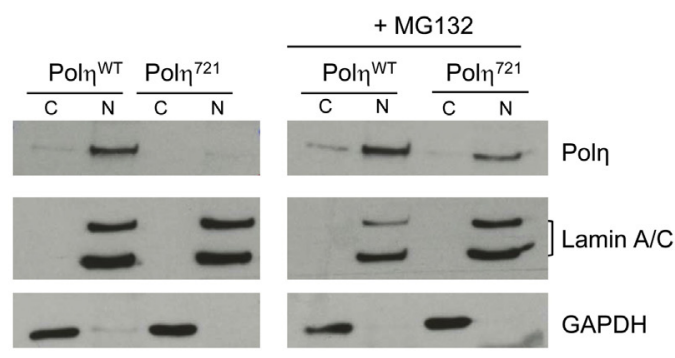

$\mathbf{E}$

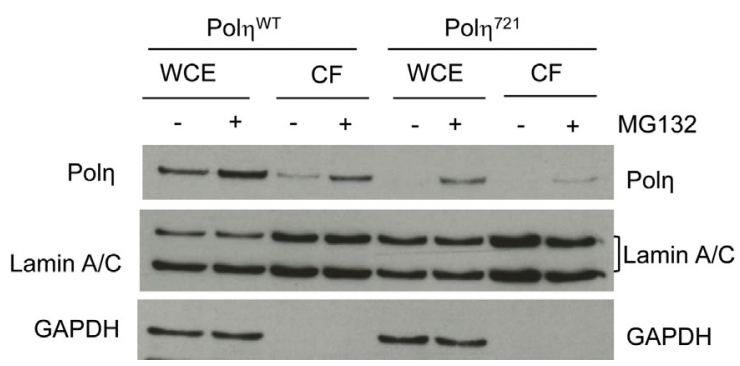

Fig. 1. Aberrant C-terminal region of Pol $\eta$ directs the protein to the degradation in the nucleus. (A) Schematic representation of human wild-type Pol $\eta$ and the mutations described in the XP-V patient (XP872VI). The different domains of the Pol $\eta$ are depicted: The active polymerase domain corresponds to the first 432 AA. The C-terminal part contains the regulation domains including the bipartite nuclear localization signal (NLS), an ubiquitin-binding Zn finger (UBZ), and a PCNA Interacting Peptide motif (PIP-box) [2,18]. In XP872VI patient, two missense mutations were found in the regulatory domain of the protein: one in the NLS (T692A) and another one on the endogenous stop codon leading a longer protein with extra 8 amino acids. (B) Detection of Pol $\eta$ level in XP872VI (XP-V) and 405VI (WT) primary fibroblasts with or without addition of $10 \mu \mathrm{M}$ MG132 for $3 \mathrm{~h}$. (C) XP30RO cells stably expressing Pol $\eta^{\mathrm{WT}}$ or POLH cDNA harboring the missense mutation on the stop codon leading to a longer protein with an extra 8 amino acids (called Pol $\eta^{721}$ ) were treated or not with MG132 for $3 \mathrm{~h}$. Protein levels of Pol $\eta^{\mathrm{WT}}$ and Pol $\eta^{721}$ were analyzed by western blot using anti-Pol $\eta$ antibody. GAPDH was used as loading control. (D) XP30RO cells stably expressing Pol $\eta^{\mathrm{WT}}$ or Pol $\eta^{721}$ were treated or not for $1 \mathrm{~h}$ with MG132. Nuclear/cytoplasmic fractionation was then performed. Cytoplasmic $(\mathrm{C})$ and nuclear $(\mathrm{N})$ extracts were analyzed by western blotting with antibodies against Poln, Lamin A/C and GAPDH. (E) XP30RO cells stably expressing Pol $\eta^{\mathrm{WT}}$ or Pol $\eta^{721}$ were treated or not for $1 \mathrm{~h}$ with MG132. Whole cell extract (WCE) and chromatin fraction (CF) were collected and analyzed by western-blotting using antibodies against Pol $\eta$, Lamin A/C and GAPDH.

Then we tested if the extra C-terminal region of Pol $\eta^{721}$ could affect its binding with mono-ubiquitinated PCNA (mUb-PCNA). To address this, cell extracts from UV-irradiated XP30RO-Pol $\eta^{721}$ cells were immunoprecipitated using anti-PCNA antibodies as previously described [20]. Again, to get a chance to observe an interaction, we treated the XP30RO-Pol $\eta^{721}$ cells with MG132 for $1 \mathrm{~h}$ before harvesting. We also used two other cell lines as control: the XP30RO-Pol $\eta^{\text {wt }}$ and the XP30RO-Pol $\eta^{\text {D652A }}$ cells which express 
A

Poln
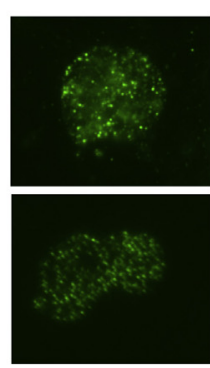

B

C
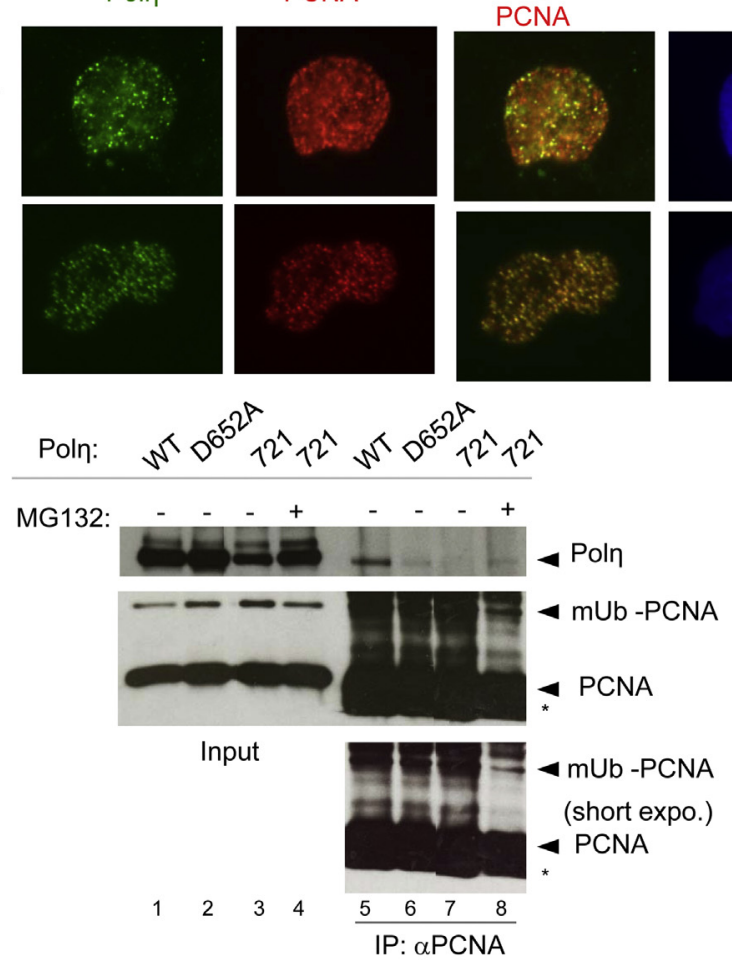

$$
\text { (a) }
$$

DAPI
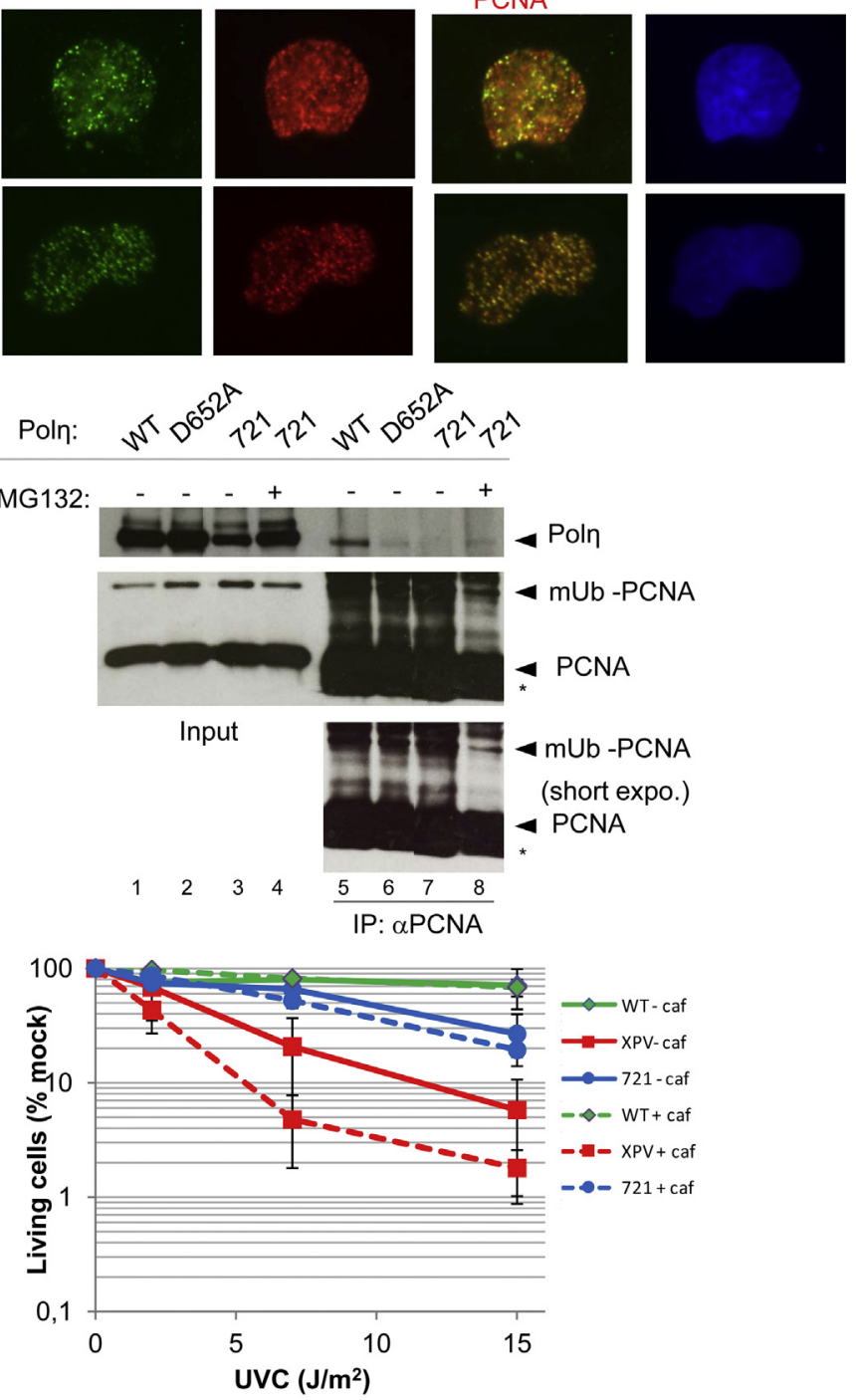

D

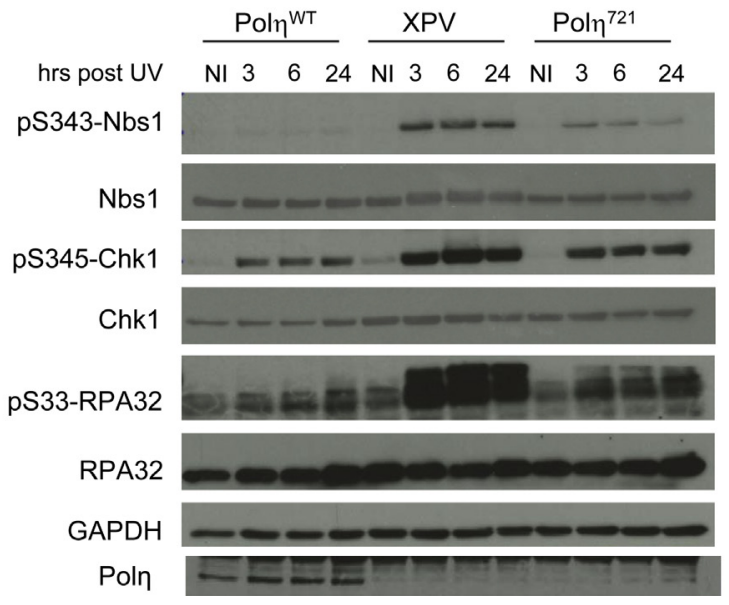

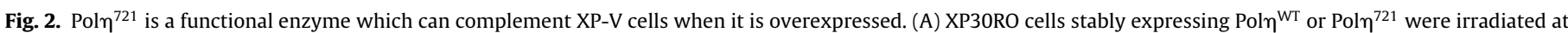

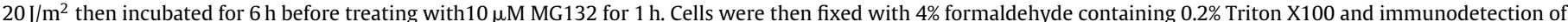

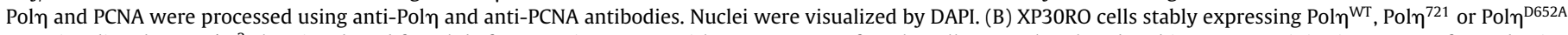

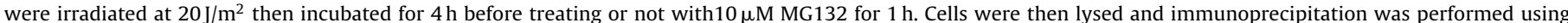

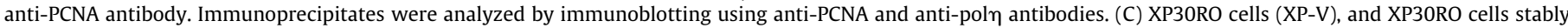

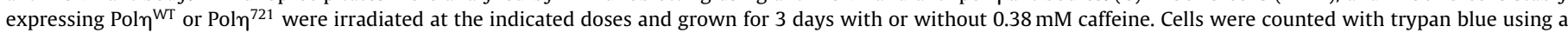

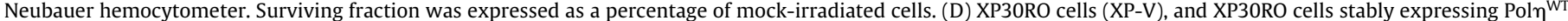

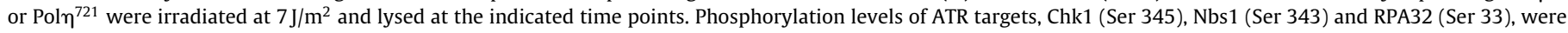
analyzed by western-blotting. 
Pol $\eta$ containing a point mutation in its UBZ domain and known to be impaired in localization to PCNA foci [7]. Western blot analysis showed that the Pol $\eta^{721}$ is able to interact with mUb-PCNA albeit at lower level than Pol $\eta^{\mathrm{wt}}$ (Fig. 2B). It has to be noted that in this experiment, we needed to treat cells with a proteasome inhibitor to stabilize Poln ${ }^{721}$. However it is known that this treatment also reduces the PCNA monoubiquitination levels [27] even during the immunoprecipitation process. This can be observed when we compare the levels of monoUb PCNA after IP in cell extracts treated or not with MG132 (Fig. 2B, lanes 7 and 8). Since we did not recover much monoUb PCNA, we did not expect to detect a reasonable amount of Pol $\eta^{721}$ in co-IP (lane 8). However we cannot rule out that the extra 8 amino acids of Pol $\eta^{721}$ directly impact on the interaction between mono-Ub PCNA and Pol $\eta$.

Next, we asked if Pol $\eta^{721}$ can complement XP-V cells given that only a residual amount of the protein is maintained in untreated $\mathrm{XP30RO-Pol} \eta^{721}$ cells. One of the characteristics of XP-V cells is their ability to be sensitized to UV with low concentrations of caffeine [1]. Hence, we compared the proliferation of cells irradiated at various UV doses and incubated in the presence or not of $0.38 \mathrm{mM}$ caffeine. This concentration increased the sensitivity of XP-V cells but had no effect on XP-V cells complemented by Pol $\eta^{\text {wt }}[6,7]$. As shown in Fig. 2C, XP-V cells expressing Pol $\eta^{721}$ were only slightly sensitive to UV and cells showed a barely sensitization by caffeine strongly suggesting that the extra 8 amino acids in C-terminal of the protein do not impair the ability of Pol $\eta^{721}$ to bypass UV-induced lesions in vivo even when the protein is present at a very low level. These results are in agreement with the restoration of normal cell cycle profiles $24 \mathrm{~h}$ after UV-irradiation in this cell line (Fig. S2C).

To confirm these results, we monitored the ATR checkpoint activation in UV-irradiated XP30RO-Pol $\eta^{712}$ cell line. Indeed, replication fork stalling at UV-induced DNA damage activates a checkpoint regulated by the ATR kinase that allows S-phase slow down and stabilization of replication forks (for review, see [4]). We have previously shown that persistent inhibition of DNA synthesis

A
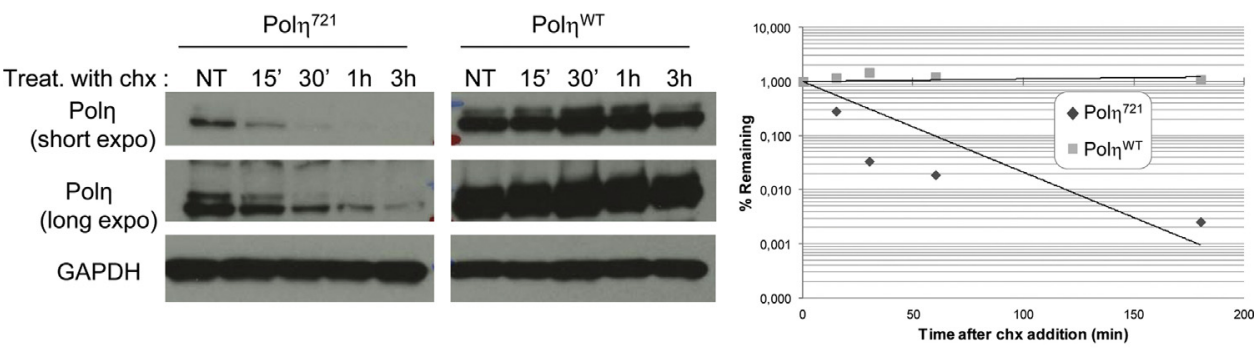

B

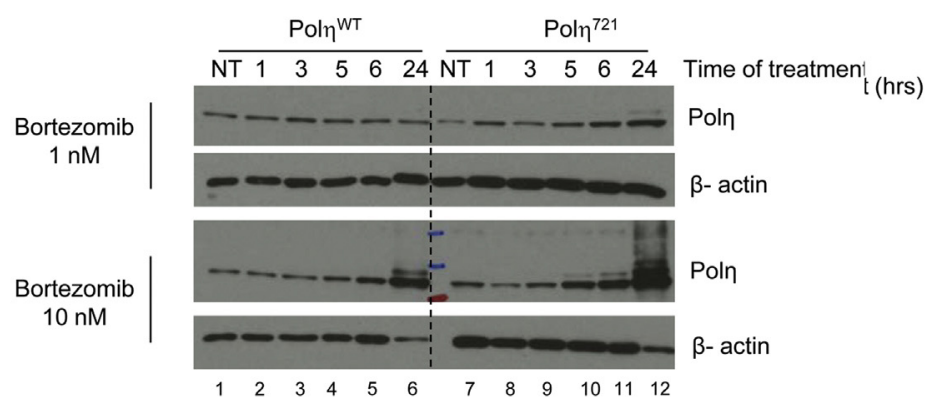

C

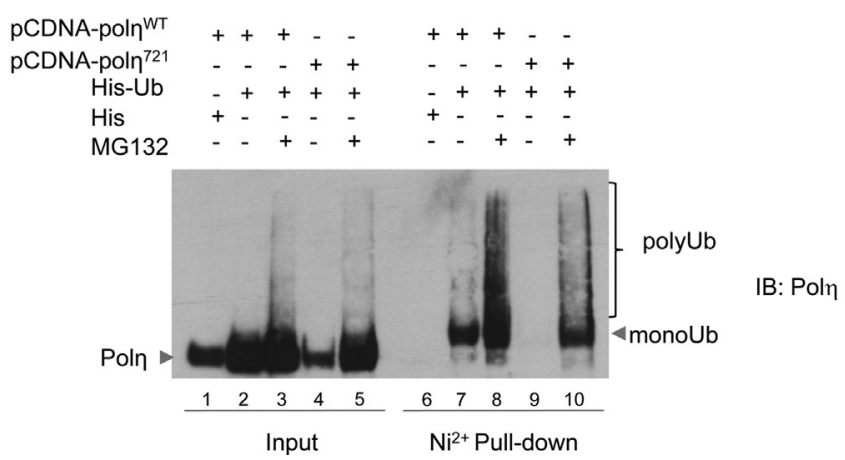

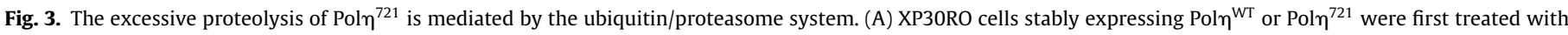

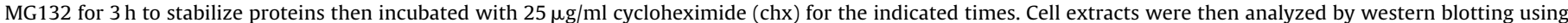

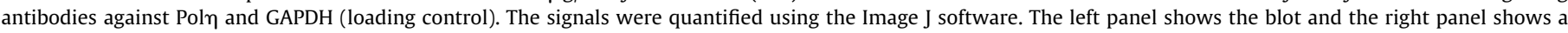

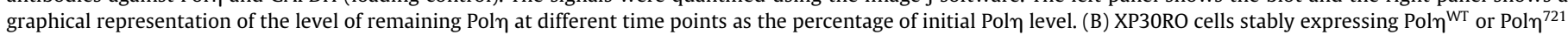

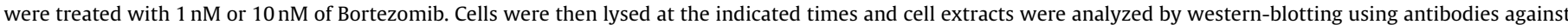

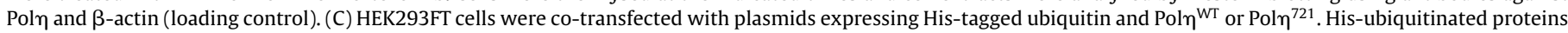
were $\mathrm{Ni}^{2+}$ pulled-down in denaturing conditions and His-Ub Pol $\eta$ proteins were immunodetected by western-blotting using antibody against Pol $\eta$. 
in UV-irradiated cells deficient in Poln was associated with a stronger and persistent activation of ATR checkpoint [6]. We analyzed the phosphorylation status of various ATR targets. These included Checkpoint Kinase 1 (Chk1), Nijmegen Breakage Syndrome 1 (Nbs1) and the 32-kDa subunit of Replication Protein A (RPA32). After $7 \mathrm{~J} / \mathrm{m}^{2}$, these proteins are more strongly phosphorylated in XP-V cells than in XP-V cells complemented with Pol $\eta^{\text {wt }}$ and phosphorylation levels remained high $24 \mathrm{~h}$ after UV exposure in the absence of Pol $\eta$ (Fig. 2D). In XP30RO-Pol $\eta^{721}$ cells, amounts of phosphorylated Chk1, Nbs1 and RPA32 were at intermediate levels after UV irradiation suggesting that in these cells UV-induced lesions can be bypassed but less efficiently than in XP30RO-Pol $\eta^{\mathrm{wt}}$ cells. This could explain why we found a slight sensitization by caffeine, known to inhibit ATR checkpoint pathway.

Altogether, our data strongly suggest that Pol $\eta^{721}$ is functional in vitro and in vivo and that the XP872VI patient displays a XP-V phenotype because the mutant protein is excessively unstable.

\subsection{The excessive proteolysis of Pol 721 is mediated by the} ubiquitin-proteasome system

We then concentrated our efforts to determine the cause of Pol $\eta^{721}$ instability. We already showed that Pol $\eta^{721}$ degradation was mediated by the proteasome (Fig. 1). We then measured the half-life of Pol $\eta^{721}$ protein by treating cells with the protein synthesis inhibitor cycloheximide (chx) and Pol $\eta$ levels were monitored at time intervals of up to $3 \mathrm{~h}$. Pol $\eta^{\mathrm{WT}}$ was stable for at least $3 \mathrm{~h}$ whereas Pol $\eta^{721}$ was found to have a half-life of $\sim 15 \mathrm{~min}$ (Fig. 3A). As shown on the graph, after $1 \mathrm{~h}$ of $\mathrm{chx}$, there was ten times less Pol $\eta^{721}$ than Pol $\eta^{\mathrm{WT}}$ (Fig. 3A, right panel). Interestingly, we also found that the clinically approved proteasome inhibitor, Bortezomib, strongly reduces the turnover of Pol $\eta^{721}$ even after a dose as low as $1 \mathrm{nM}$ (Fig. 3B). We also tested if this proteasome inhibitor was able to stabilize the Pol $\eta$ missense variant in primary XP872VI fibroblasts by treating XP-V and 405VI cells with 10 and $100 \mathrm{nM}$ Bortezomib for $3 \mathrm{~h}$. As observed after MG132 treatment, Bortezomib slightly restores the variant Poln protein levels (Fig. S3).

It has been shown that Pol $\eta$ degradation can be targeted by different mechanisms in human cells. Indeed the E3 ligase Pirh2 promotes Pol $\eta$ degradation in an ubiquitin-independent manner [16] while MDM2 can also serve as an E3 ligase for Poln polyubiquitination and proteosomal degradation in response to ultraviolet irradiation [17]. We observed that incubation of cells with $10 \mathrm{nM}$ Bortezomib leads to the accumulation of slower migrating forms of Pol $\eta^{721}$ (Fig. 3B, lane 12) which seem to be poly-ubiquitin chains strongly suggesting that $P o l \eta^{721}$ is polyubiquitinated before degradation. To confirm this, we carried out a $\mathrm{Ni}^{2+}$ pull-down in 293FT cells over-expressing the Pol $\eta$ mutant and His-ubiquitin (His-Ub) and showed that Pol $\eta^{721}$ as Pol $\eta^{\text {wt }}$ is efficiently poly-ubiquitinated after MG132 treatment (Fig. 3C, lane 10). Collectively, these results demonstrate that Pol $\eta^{721}$ is degraded by the proteasome in a ubiquitin-dependent manner.

\subsection{The bipartite NLS is partially responsible of the excessive Pol ${ }^{721}$ proteolysis}

We then attempted to identify the amino acid sequence in the C-terminal part of Pol $\eta$ mutant critical for this aberrant proteolysis. We first asked if the last 80 amino-acids of Pol $\eta^{721}$ (spanning the NLS and PIP motifs of the wild type sequence + the extra 8 aminoacids) are capable of destabilizing a completely unrelated protein. To do that, we constructed a chimeric polypeptide in which the last 80 amino acids of Pol $\eta^{721}$ (or the last 70 amino-acids of the wild-type Pol $\eta^{\mathrm{wt}}$ ) were fused to the C-terminal end of the eGFP reporter (denoted GFP-Pol $\eta 80$ and GFP-Pol $\eta$ 70, respectively) and monitored the stability of the fusion proteins. As shown in Fig. 4B,
GFP-Pol $\eta 80$ exhibited a strong instability as compared to GFP-Poln $70(1 \mathrm{~h} v s 3 \mathrm{~h})$. As illustrated on the graph, after $3 \mathrm{~h}$ of chx, there

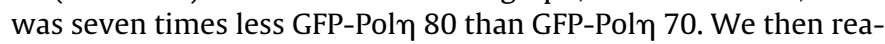
soned that the extra 8 amino-acids might act as a degron. To test this notion, we examined the stability of fusion proteins containing point mutations in the extra C-terminal part of Pol $\eta^{721}$ (Fig. S3). All the mutants did not show any increase of protein stability unless cells were treated with MG132. Even mutations on the 8 amino acids (eGFP-Poln $808 \mathrm{~A}$ ) cannot stabilize the fusion protein (Fig. 4C and Fig. S4). These results indicate that the extra C-terminal part of Pol $\eta^{721}$ does not function as a degron. From these data we assumed that the extra 8 amino-acids have changed the conformation of the C-terminal part of Poln exposing the protein to the ubiquitin/proteasome system.

To gain insight into the specific residues in Pol $\eta^{721}$ that are important for its proteolysis, we constructed a series of truncated Pol $\eta$ mutants fused to GFP reporter and analyzed their stability in cells (Fig. 4D). One construct contains the last 40 amino-acids spanning the NLS, PIP and the extra C-terminus of Pol $\eta^{721}$ (GFP-Pol $\eta$ 40), another one contains the PIP and the extra C-terminus (GFP-Poln 20), or only the extra C-terminus (GFP-Poln extra C-term). Cells expressing GFP constructs were treated or not with MG132 for $3 \mathrm{~h}$ and fusion protein levels were analyzed by western blot. As shown in Fig. 4E, GFP-Poln extra C-term was as stable as the GFP alone confirming that this motif does not act as a degron. Interestingly we found that GFP-Poln 20 lacking the bipartite NLS was less destabilized than GFP-Pol $\eta 40$ suggesting that the Pol $\eta$ NLS can participate in the proteolysis of Pol $\eta^{721}$. Alternatively it is possible that in the absence of NLS, the fusion protein is not driven into the nucleus limiting its degradation. To rule out this possibility, we examined the half life of each construct as well as that of a new construct in which we replaced the bipartite NLS by a NLS peptide which derives from the SV40 Large T antigen (GFP-NLS AgT Pol $\eta$ 40). Cells expressing GFP constructs were treated with chx till $7 \mathrm{~h}$ and fusion protein amounts were analyzed at different time points by western blot (Fig. 4F, left panel). As expected the half life of GFP-Pol $\eta 40$ was much shorter than that of GFP alone. Indeed GFP was stable for at least $7 \mathrm{~h}$ whereas GFP-Poln 40 was found to have a half-life $<2 \mathrm{~h}$ (Fig. 4F, right panel). Importantly, by replacing the bipartite NLS by $\mathrm{T}$-Ag NLS peptide, we increased the stability of the chimeric protein with a half life similar to that of GFP-Poln 20 lacking the bipartite NLS (about $3 \mathrm{~h}$, Fig. 4F, right panel). These results suggested that the bipartite NLS of Pol $\eta$ is directly or indirectly involved in the degradation of Pol $\eta^{721}$.

\subsection{The four mono-ubiquitination sites of Poln are partially involved in the proteolysis of Poln 721}

Previous works reported that in physiological condition, a fraction of Poln is mono-ubiquitinated in human cells and that mono-ubiquitination of Pol $\eta$ occurs C-terminal to its UBZ domain at one of the four lysine residues available in this region (Fig. 5A). The hypothesis is that the attachment of Ub to the C-terminus of Poln, by introducing an intramolecular interaction with the UBZ motif, prevents this domain from being engaged in other interactions and also sterically masks the region, hiding it from PCNA [3]. Interestingly, three out of four mono-ubiquitination sites are in the NLS sequence (e.g. K682, K686, K694). Because we found that the bipartite NLS was implicated in the proteolysis of Pol $\eta^{721}$, we asked if these lysine residues were required for its excessive destabilization. To this aim, we generated four different mutants of Pol $\eta^{721}$ which contain one, two, three or four $\mathrm{K}$ to R substitutions (Fig. 5A). XP-V cells expressing these constructs were or not treated with MG132 and protein levels were analyzed by western blotting. We observed that the more Pol $\eta^{721}$ contains K/R substitutions, the more the protein was stable without addition of proteasome inhibitor (Fig. 5B) 
A

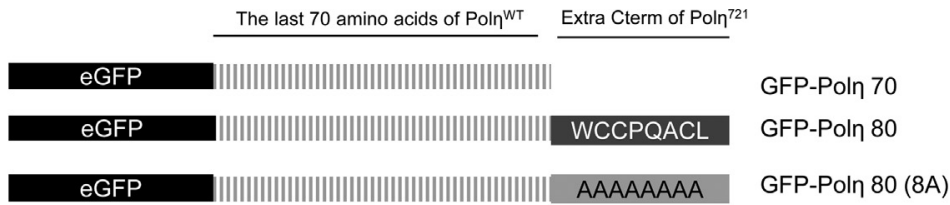

B

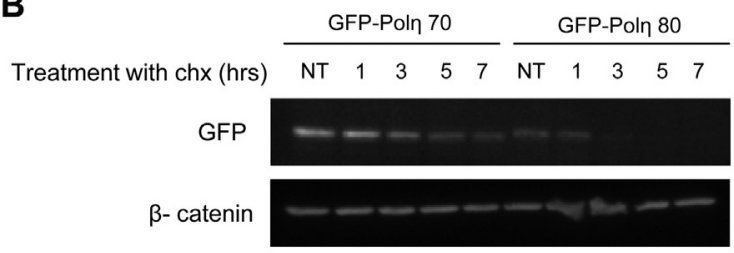

C
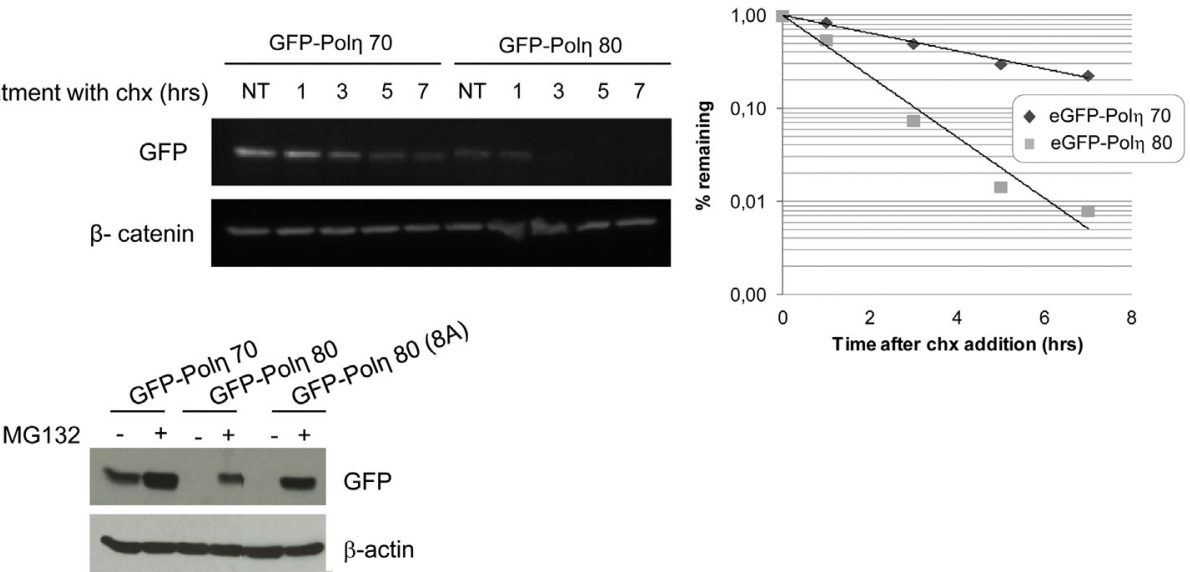

D

\begin{tabular}{|c|c|c|c|c}
\hline eGFP & $\|$ NLS\$ $\|$ & PIP2 & WCCPQACL & GFP-Poln 40 \\
\hline eGFP & Ag-T NLS & PIP2 & WCCPQACL & GFP- NLS AgT Poln 40 \\
\hline eGFP & PIP2 & WCCPQACL & GFP-Poln 20 \\
\hline eGFP & WCCPQACL & GFP-Poln extra C-term
\end{tabular}

$\mathbf{E}$

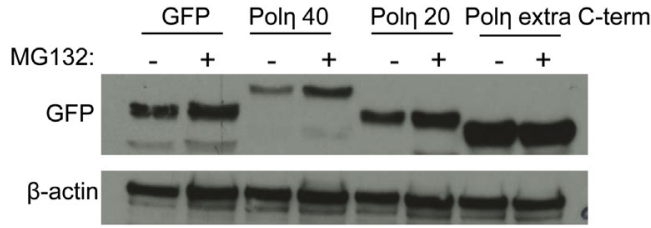

$\mathbf{F}$

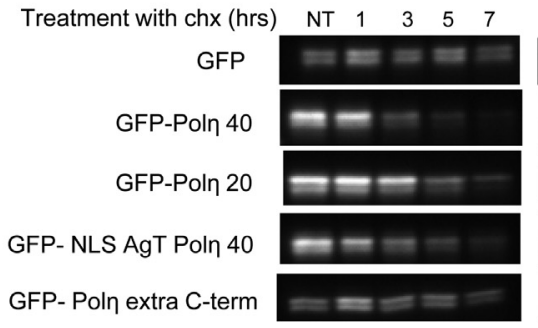

IB: GFP

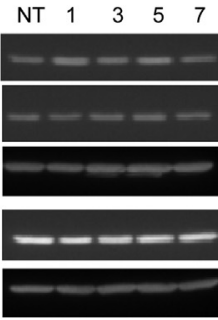

IB: $\beta$ - actin

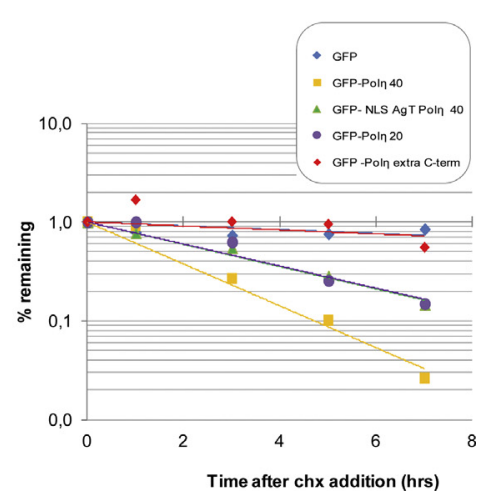

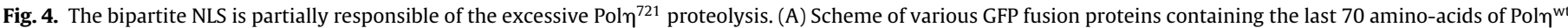

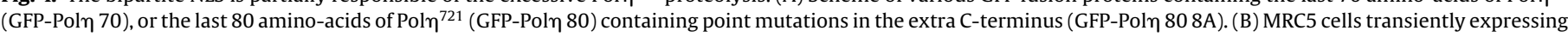

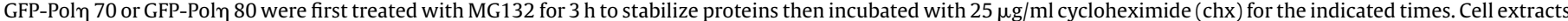

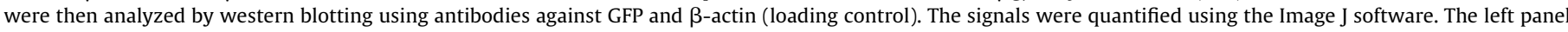

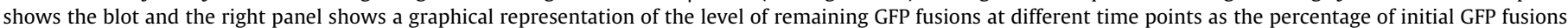

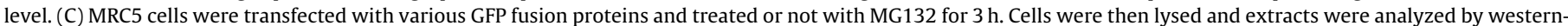

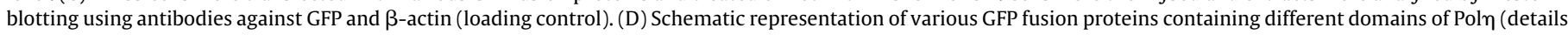

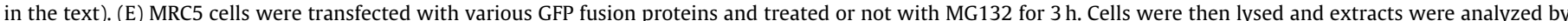

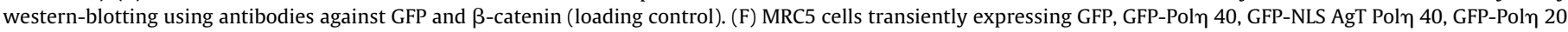
and GFP-Pol $\eta$ extra C-term were analyzed as described in (B).

These data are consistent with the involvement of the bipartite NLS in the proteolysis of Pol $\eta^{721}$.

It was reported that when all four lysines were mutated to arginines (Poln 4K/R), no ubiquitinated species of Pol $\eta$ were detectable in whole cell extracts [3]. We confirmed this observation with two independent clones (Fig. 5C, lanes 3 and 4). In contrast, the mutant Pol $\eta^{721} 4 \mathrm{~K} / \mathrm{R}$ remained mono-ubiquitinated (Fig. 5C, lane 5) reinforcing the hypothesis that the extra 8 amino acids sequence modifies the conformation of the C-terminus of Pol $\eta^{721}$ allowing the ubiquitination of other lysines in close proximity of (or in) the 
A

Figure 5

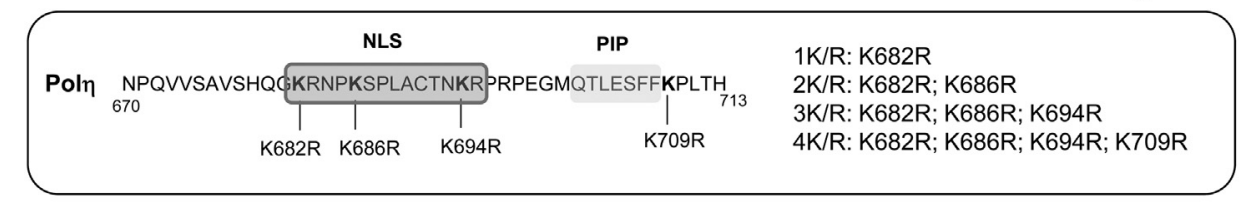

B

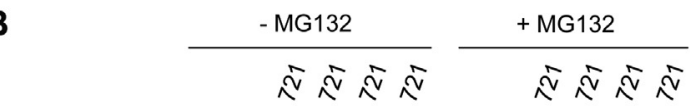

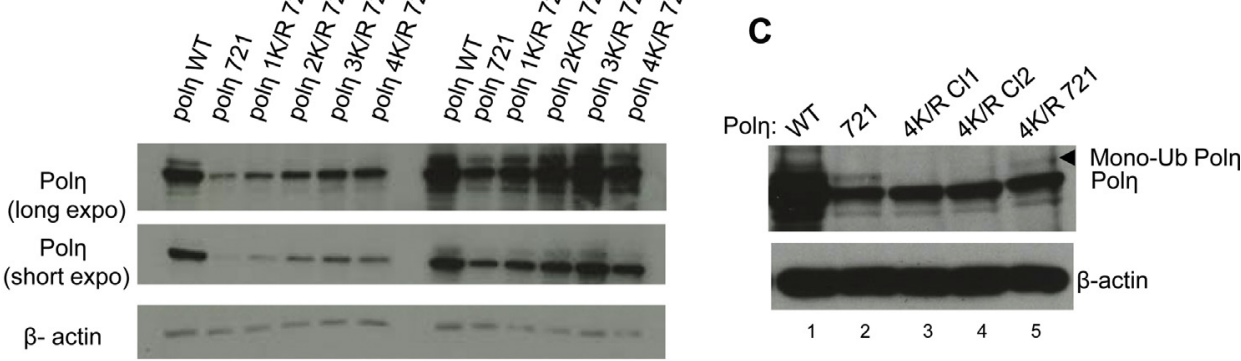

D
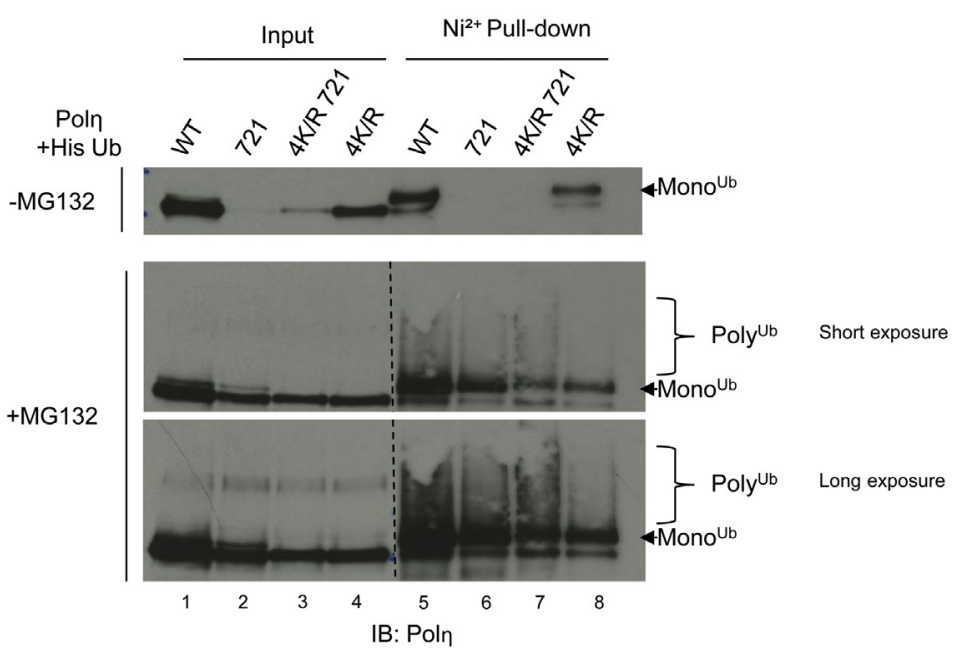

E

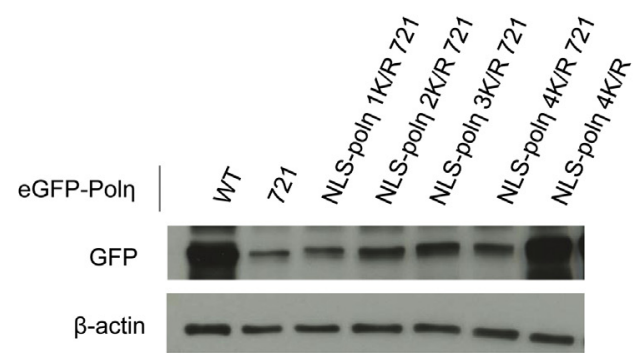

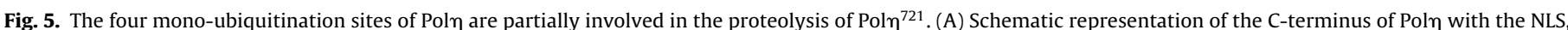

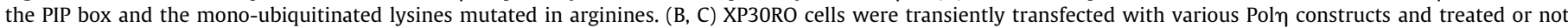

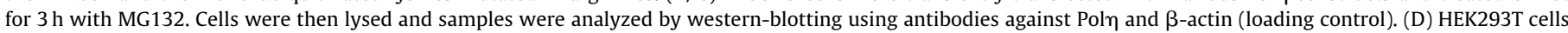

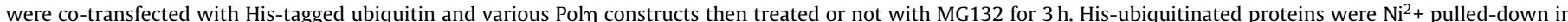

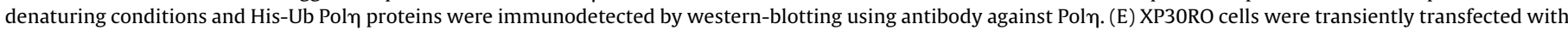

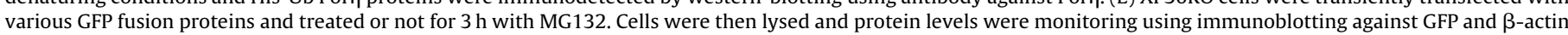
(loading control).

UBZ domain. We then checked if this Pol $\eta^{721} 4 \mathrm{~K} / \mathrm{R}$ mutant was also degraded in a ubiquitin-dependent manner. To address this, we performed a $\mathrm{Ni}^{2+}$ pull-down from cells over-expressing different Pol $\eta$ mutants as well as His-Ub and analyzed the ubiquitinated species of Pol $\eta$ by western blot. Unexpectedly we observed monoubiquitinated forms of Pol $\eta^{\mathrm{wt}} 4 \mathrm{~K} / \mathrm{R}$ after pull down (Fig. 5D, lane 8). Western blot analysis also revealed that in contrast to Pol $\eta^{\mathrm{wt}}$
4K/R, Pol $\eta^{721} 4 \mathrm{~K} / \mathrm{R}$ was efficiently poly-ubiquitinated after MG132 treatment (Fig. 5D, lane $7 v$ s lane 8 ). These data suggest that excessive proteolysis of Pol $\eta^{721}$ involves additional lysines located in the C-terminal part of the protein which are targeted for proteosomal degradation.

Because three out of four lysines were within the NLS, we reasoned that the partial stabilization of the K/R mutants could result 
A

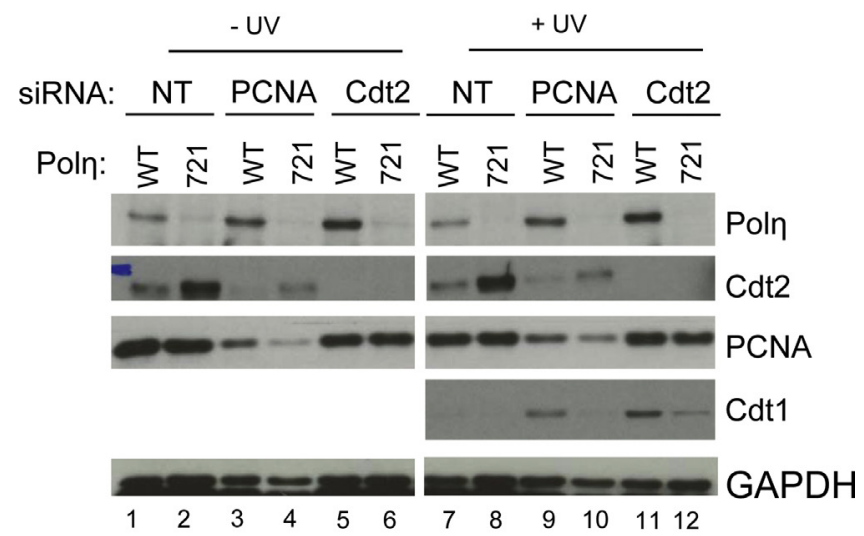

B

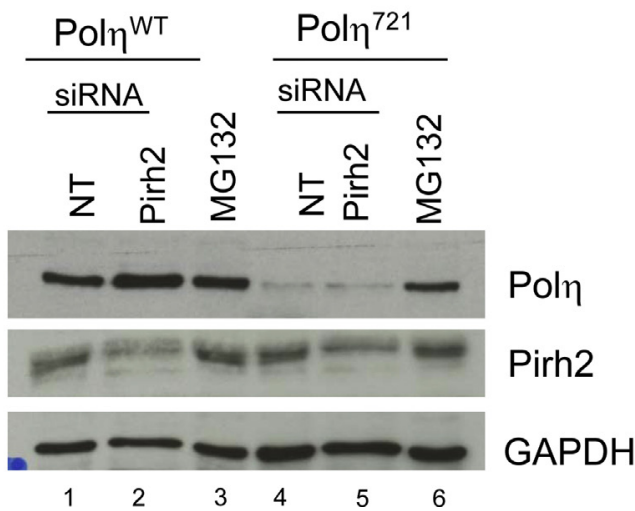

Fig. 6. The E3 ligases CRL4 ${ }^{\mathrm{Cdt} 2}$ and Pirh 2 are not involved in the excessive degradation Pol $\eta^{721}$. (A) XP30RO cells stably expressing Pol $\eta^{\mathrm{WT}}$ or Pol $\eta^{721}$ were transfected with non-targeted siRNAs (NT) or siRNAs targeting PCNA or CRL4 ${ }^{\text {Cdt2 }}$. Seventytwo hours later, cells were irradiated or not at $20 \mathrm{~J} / \mathrm{m}^{2}$. Levels of Poln, CRL4 ${ }^{\mathrm{Cdt} 2}$, PCNA, Cdt1 and GAPDH (loading control) were monitored with specific antibodies. (B) XP30RO cells stably expressing Pol $\eta^{\mathrm{WT}}$ or Pol $\eta^{721}$ were transfected with nontargeted siRNAs (NT) or siRNAs targeting Pirh2 for $72 \mathrm{~h}$ later or treated with MG132 for $3 \mathrm{~h}$ prior to harvest. Proteins were analyzed by western-blotting using antibodies against Pol $\eta$, Pirh2 and GAPDH (loading control).

from an incorrect nuclear localization leading to an inefficient proteolysis. We then decided to introduce an exogenous SV40 large T-Ag NLS at the N-terminus of the Pol $\eta$ in fusion with the GFP. As shown in Fig. 5E, these mutants remained stabilized even when they localized into the nucleus.

Altogether, these results suggest that the extra 8 amino-acids of Pol $\eta^{721}$ induce a conformational change of the C-terminal region of

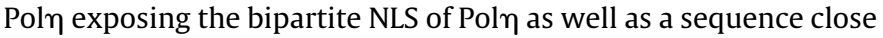
to the UBZ to the ubiquitin/proteasome system.

\subsection{The E3 ligases CRL4 ${ }^{\text {Cdt }}{ }^{2}$ and Pirh2 are not involved in the proteolysis of Pol $\eta^{721}$}

In C. elegans, the GEI-17 SUMO E3 ligase protects PolH protein from degradation mediated by CRL4 ${ }^{\text {Cdt2 }}$ pathway [21]. In human cells the four lysines located in the C-terminus of Poln were found to be mono-ubiquitinated by Pirh2 to inhibit the ability of Pol $\eta$ to interact with PCNA [15].

In an attempt to identify the E3 ligase involved in the proteolysis of Pol $\eta^{721}$ we first analyzed the levels of the TLS polymerase after down-regulation of CRL4 ${ }^{\text {Cdt2 } 2}$. We also monitored the protein levels of cdt 1 as positive control since it is known that knock-down of PCNA or CRL4 ${ }^{\text {Cdt2 }}$ increased the stability of Cdt1, especially after UV irradiation [10]. As shown in Fig. 6A (lanes 6 and 12), efficient down-regulation of the E3 ligase CRL4 ${ }^{\text {Cdt2 }}$ does not stabilize Pol $\eta^{721}$ while it stabilizes cdt1 (Fig. 6A, lanes 11 and 12). We then explored the impact of down-regulation of the E3 ligase Pirh2 on Pol $\eta^{721}$ stability. While we detected an increase of Pol $\eta^{\text {wt }}$ levels after Pirh2 depletion (Fig. 6B, lane 2) we did not observe any Pol $\eta^{721}$ stabilization (Fig. 6B, lane 5). Altogether these results revealed that CRL4 ${ }^{\text {Cdt2 }}$ and Pirh2 are not involved in the proteolysis of Pol $\eta^{721}$.

\section{Discussion}

We recently reported the case of a XP-V patient (XP872VI) with "mild symptoms" harboring two missense mutations at the homozygous state: one mutation on the bipartite NLS (T692A) and a second mutation on the stop codon (X714W) leading to a longer protein with a extra 8 amino acids (Fig. 1A). The Poln missense variant was barely detectable by western blot suggesting that it is extremely unstable [26]. Several studies reported that missense mutations affect the stability of Poln regardless of the location of the mutation in the protein structure [12,26,33]. As these Poln missense variants are extremely unstable and nearly undetectable, a definitive measure of their functional deficit in cells has not been fully explored. Indeed, it is unknown if the patients exhibit a XP-V phenotype because the mutant protein is functional but excessively unstable or if the protein is not functional and the residual amount is unable to bypass UV-induced DNA damage. This question has to be solved if we want to propose a therapeutic approach to treating tumors (or preventing cancer progression) in XP-V patients harboring missense mutations in POLH gene.

In the present study we have characterized the Pol $\eta$ missense variant, Pol $\eta^{721}$ at the cellular and molecular level by generating a POLH cDNA harboring the missense mutation on the stop codon leading to a longer protein with an extra 8 amino acids and by stably expressing this variant in XP-V fibroblasts. We first found that Pol $\eta^{721}$ is excessively degraded by $20 S$ proteasome in a ubiquitin-dependent manner. Blocking degradation through chemical inhibition of the proteasome restored levels and function to Pol $\eta^{721}$. Indeed, we found that stabilized Pol $\eta^{721}$ localizes in the replication factories after UV-irradiation and interacts with mUbPCNA albeit at lower level than Pol $\eta^{\mathrm{wt}}$.

Moreover, XP-V cells overexpressing Pol $\eta^{721}$ show only a slight sensitivity to UV compared to XP-V cells. Finally, in our in vitro assay, Pol $\eta^{721}$ is able to bypass CPD lesions albeit less efficiently than Pol $\eta^{\text {wt }}$. From these results we concluded that Pol $\eta^{721}$ is functional and that the XP872VI patient displays a XP-V phenotype because the mutant protein is excessively degraded. This is consistent with the fact that the patient has only "mild symptoms".

Interestingly we showed that the clinically approved proteasome inhibitor Bortezomib restores the levels of Pol $\eta^{721}$ suggesting that this may be a viable therapeutic approach to preventing tumor development in certain XP-V patients. However general inhibition of proteasome activity could be very toxic, particularly in the long-term treatment. It is noteworthy that $20 \mathrm{~S}$ proteasome inhibitors (Bortezomib, Carfilzomib...) are already used for treating multiple myeloma (for review [9,31]). Indeed, Bortezomib is currently used in combination with an alkylating agent Melphalan which induces alkylation of guanine and adenine as well as interstrand DNA crosslinks. However resistances to Melphalan have been observed in multiple myeloma cells [9]. Given that Pol $\eta$ is able to bypass alkylated DNA lesions [14] and that Bortezomib stabilizes the TLS polymerase, the combination of the two drugs might explain development of drug resistance.

By performing a comprehensive analysis of the amino acid sequence in the C-terminal part of Pol $\eta^{721}$ critical for its excessive proteolysis, we found that the extra 8 amino acids do not act as a degron but rather might induce a conformational change of the $C$ terminal of Poln exposing the protein to the ubiquitin/proteasome 
system. Previous works reported that a fraction of human Pol $\eta$ is mono-ubiquitinated at one of the four lysine residues located in the C-terminal part of the protein (K682, K686, K694, and K709) to inhibit its interaction with PCNA $[3,15]$. Three out of the four ubiquitination sites are present in the bipartite NLS of Poln. We found that these lysines were implicated in the degradation of Pol $\eta^{721}$ since mutations to arginines increase the level of Pol $\eta$ variant. However, it did not reach the Pol $\eta^{\text {wt }}$ level indicating that proteolysis of Pol $\eta^{721}$ involves additional lysine residues, apart the four mono-ubiquitinated sites already identified. Consistent with this hypothesis, we observed that in contrast to Pol $\eta^{\mathrm{wt}} 4 \mathrm{~K} / \mathrm{R}$, the mutant Pol $\eta^{721} 4 \mathrm{~K} / \mathrm{R}$ is still poly-ubiquitinated then degraded by the $20 \mathrm{~S}$ proteasome.

So far three E3 ligases (e.g. CRL4 ${ }^{\mathrm{cdt} 2}$, Pirh2 and mdm2) have been involved in the ubiquitination and/or degradation of Poln $[16,17,21]$. In this study we report that the degradation of Pol $\eta^{721}$ is independent of CRL4 ${ }^{\text {cdt2 }}$ and Pirh2. Since Mdm2 has been involved in the ubiquitination and degradation of Poln after UV irradiation, it is unlikely that this E3 ligase is involved in the proteolysis of the Pol $\eta^{721}$ observed in unstressed cells [17]. Therefore it is tempting to assume that another E3 ligase is implicated in this degradation. Interestingly, it has been reported that the chaperone HSP90 recognizes Pol $\eta$ as a client and facilitates its accumulation at fork stalling sites through regulation of its stability/solubility [30]. Hsp90 promotes proper folding of newly synthesized proteins into mature forms, and regulates stability, and protein interactions of mature clients. If the native state is unattainable, misfolded proteins are targeted for degradation via the ubiquitin-proteasome system. The specificity of this proteolysis is generally provided by E3 ubiquitin ligases [22]. One of the best characterized proteins that links molecular chaperones with protein degradation is the $\mathrm{E} 3$ ubiquitin ligase CHIP (C-terminus of Hsc70 Interacting Protein) which is a U-box E3 enzyme belonging to a new category of E3 enzyme responsible for quality control of cellular proteins [5]. Therefore, we believe that the chaperone associated ubiquitin ligase, CHIP is an attractive candidate for degrading Pol $\eta^{721}$. This hypothesis is currently under investigation.

\section{Funding}

The P.L.K. lab is supported by La Ligue Nationale contre le Cancer (Equipe labellisée). This work was supported by grants from Institut National du Cancer (INCa) PLBio-2010 and PLBio2012. S.A.S. received support from the Ministère de l'Enseignement Supérieur et de la Recherche and the Foundation Association pour la Recherche sur le Cancer. E.D. received fellowship from INCa PLBio2010.

\section{Conflict of interest statement}

The authors declare that there are no conflicts of interest.

\section{Acknowledgments}

We are grateful to JE Cleaver (UCSF, CA) for supplying XP30RO cells and Robert Fuchs (CRCM, France) for providing singlestranded plasmids containing a CPD lesion (pUC-CPD.ss). We thank Florian Dupuy for technical assistance and members of the Kannouche lab as well as Said Aoufouchi for helpful discussions.

\section{Appendix A. Supplementary data}

Supplementary data associated with this article can be found, in the online version, at http://dx.doi.org/10.1016/ j.dnarep.2015.02.017.

\section{References}

[1] C.F. Arlett, S.A. Harcourt, B.C. Broughton, The influence of caffeine on cell survival in excision-proficient and excision-deficient xeroderma pigmentosum and normal human cell strains following ultraviolet-light irradiation, Mutat. Res. 33 (1975) 341-346.

[2] M. Bienko, C.M. Green, N. Crosetto, F. Rudolf, G. Zapart, B. Coull, P. Kannouche, G. Wider, M. Peter, A.R. Lehmann, K. Hofmann, I. Dikic, Ubiquitin-binding domains in Y-family polymerases regulate translesion synthesis, Science 310 (2005) $1821-1824$

[3] M. Bienko, C.M. Green, S. Sabbioneda, N. Crosetto, I. Matic, R.G. Hibbert, T. Begovic, A. Niimi, M. Mann, A.R. Lehmann, I. Dikic, Regulation of translesion synthesis DNA polymerase eta by monoubiquitination, Mol. Cell 37 (2010) 396-407.

[4] K.A. Cimprich, D. Cortez, ATR: an essential regulator of genome integrity, Nat. Rev. Mol. Cell Biol. 9 (2008) 616-627.

[5] P. Connell, C.A. Ballinger, J. Jiang, Y. Wu, L.J. Thompson, J. Hohfeld, C. Patterson, The co-chaperone CHIP regulates protein triage decisions mediated by heatshock proteins, Nat. Cell Biol. 3 (2001) 93-96.

[6] E. Despras, F. Daboussi, O. Hyrien, K. Marheineke, P.L. Kannouche, ATR/Chk1 pathway is essential for resumption of DNA synthesis and cell survival in UVirradiated XP variant cells, Hum. Mol. Genet. 19 (2010) 1690-1701.

[7] E. Despras, N. Delrieu, C. Garandeau, S. Ahmed-Seghir, P.L. Kannouche, Regulation of the specialized DNA polymerase eta: revisiting the biological relevance of its PCNA- and ubiquitin-binding motifs, Environ. Mol. Mutagen. 53 (2012) $752-765$.

[8] E.C. Friedberg, A.R. Lehmann, R.P. Fuchs, Trading places: how do DNA polymerases switch during translesion DNA synthesis? Mol. Cell 18 (2005) 499-505.

[9] C. Gourzones-Dmitriev, A. Kassambara, S. Sahota, T. Reme, J. Moreaux, P. Bourquard, D. Hose, P. Pasero, A. Constantinou, B. Klein, DNA repair pathways in human multiple myeloma: role in oncogenesis and potential targets for treatment, Cell Cycle 12 (2013) 2760-2773.

[10] C.G. Havens, J.C. Walter, Mechanism of CRL4(Cdt2), a PCNA-dependent E3 ubiquitin ligase, Genes Dev. 25 (2011) 1568-1582.

[11] C. Hoege, B. Pfander, G.L. Moldovan, G. Pyrowolakis, S. Jentsch, RAD6dependent DNA repair is linked to modification of PCNA by ubiquitin and SUMO, Nature 419 (2002) 135-141.

[12] T. Itoh, S. Linn, R. Kamide, H. Tokushige, N. Katori, Y. Hosaka, M. Yamaizumi, Xeroderma pigmentosum variant heterozygotes show reduced levels of recovery of replicative DNA synthesis in the presence of caffeine after ultraviolet irradiation, J. Invest. Dermatol. 115 (2000) 981-985.

[13] R.E. Johnson, S. Prakash, L. Prakash, Efficient bypass of a thymine-thymine dimer by yeast DNA polymerase, Poleta, Science 283 (1999) 1001-1004.

[14] R.E. Johnson, S.L. Yu, S. Prakash, L. Prakash, A role for yeast and human translesion synthesis DNA polymerases in promoting replication through 3-methyl adenine, Mol. Cell. Biol. 27 (2007) 7198-7205.

[15] Y.S. Jung, A. Hakem, R. Hakem, X. Chen, Pirh2 E3 ubiquitin ligase monoubiquitinates DNA polymerase eta to suppress translesion DNA synthesis, Mol. Cell. Biol. 31 (2011) 3997-4006.

[16] Y.S. Jung, G. Liu, X. Chen, Pirh2 E3 ubiquitin ligase targets DNA polymerase eta for $20 \mathrm{~S}$ proteasomal degradation, Mol. Cell. Biol. 30 (2010) 1041-1048

[17] Y.S. Jung, Y. Qian, X. Chen, DNA polymerase eta is targeted by Mdm2 for polyubiquitination and proteasomal degradation in response to ultraviolet irradiation, DNA Repair (Amst.) 11 (2012) 177-184.

[18] P. Kannouche, B.C. Broughton, M. Volker, F. Hanaoka, L.H. Mullenders, A.R. Lehmann, Domain structure, localization, and function of DNA polymerase eta, defective in xeroderma pigmentosum variant cells, Genes Dev. 15 (2001) $158-172$.

[19] P. Kannouche, A.R. Fernandez de Henestrosa, B. Coull, A.E. Vidal, C. Gray, D. Zicha, R. Woodgate, A.R. Lehmann, Localization of DNA polymerases eta and iota to the replication machinery is tightly co-ordinated in human cells, EMBO J. 22 (2003) 1223-1233.

[20] P.L. Kannouche, J. Wing, A.R. Lehmann, Interaction of human DNA polymerase eta with monoubiquitinated PCNA: a possible mechanism for the polymerase switch in response to DNA damage, Mol. Cell 14 (2004) 491-500.

[21] S.H. Kim, W.M. Michael, Regulated proteolysis of DNA polymerase eta during the DNA-damage response in C. elegans, Mol. Cell 32 (2008) 757-766.

[22] F. Kriegenburg, L. Ellgaard, R. Hartmann-Petersen, Molecular chaperones in targeting misfolded proteins for ubiquitin-dependent degradation, FEBS J. 279 (2012) 532-542.

[23] A. Lehmann, S. Kirk-Bell, C.F. Arlett, M.C. Paterson, P.H.M. Lohman, E.A. de Weerd-Kastelein, D. Bootsma, Xeroderma pigmentosum cells with normal levels of excision repair have a defect in DNA synthesis after UV-irradiation, Proc. Natl. Acad. Sci. U.S.A. 72 (1975) 219-223.

[24] C. Masutani, M. Araki, A. Yamada, R. Kusumoto, T. Nogimori, T. Maekawa, S, Iwai, F. Hanaoka, Xeroderma pigmentosum variant (XP-V) correcting protein from HeLa cells has a thymine dimer bypass DNA polymerase activity, EMBO J. 18 (1999) 3491-3501.

[25] T. Ogi, P. Kannouche, A.R. Lehmann, Localisation of human Y-family DNA polymerase kappa: relationship to PCNA foci, J. Cell Sci. 118 (2005) 129-136.

[26] K. Opletalova, A. Bourillon, W. Yang, C. Pouvelle, J. Armier, E. Despras, M Ludovic, C. Mateus, C. Robert, P. Kannouche, N. Soufir, A. Sarasin, Correlation of phenotype/genotype in a cohort of 23 xeroderma pigmentosum-variant 
patients reveals 12 new disease-causing POLH mutations, Hum. Mutat. 35 (2014) 117-128.

[27] B.S. Plosky, A.E. Vidal, A.R. Fernandez de Henestrosa, M.P. McLenigan, J.P. McDonald, S. Mead, R. Woodgate, Controlling the subcellular localization of DNA polymerases iota and eta via interactions with ubiquitin, EMBOJ.25(2006) 2847-2855.

[28] J.E. Sale, Translesion DNA synthesis and mutagenesis in eukaryotes, Cold Spring Harb. Perspect. Biol. 5 (2013) a012708.

[29] V. Schmutz, R. Janel-Bintz, J. Wagner, D. Biard, N. Shiomi, R.P. Fuchs, A.M. Cordonnier, Role of the ubiquitin-binding domain of Poleta in Rad18-independent translesion DNA synthesis in human cell extracts, Nucleic Acids Res. 38 (2010) 6456-6465.

[30] T. Sekimoto, T. Oda, F.M. Pozo, Y. Murakumo, C. Masutani, F. Hanaoka, T. Yamashita, The molecular chaperone Hsp90 regulates accumulation of DNA polymerase eta at replication stalling sites in UV-irradiated cells, Mol. Cell 37 (2010) 79-89.

[31] M. Shen, S. Schmitt, D. Buac, Q.P. Dou, Targeting the ubiquitin-proteasome system for cancer therapy, Expert Opin. Ther. Targets 17 (2013) 1091-1108.
[32] A. Stary, P. Kannouche, A.R. Lehmann, A. Sarasin, Role of DNA polymerase eta in the UV mutation spectrum in human cells, J. Biol. Chem. 278 (2003) $18767-18775$.

[33] M. Tanioka, T. Masaki, R. Ono, T. Nagano, E. Otoshi-Honda, Y. Matsumura, M. Takigawa, H. Inui, Y. Miyachi, S. Moriwaki, C. Nishigori, Molecular analysis of DNA polymerase eta gene in Japanese patients diagnosed as xeroderma pigmentosum variant type, J. Invest. Dermatol. 127 (2007) 1745-1751.

[34] A. Tissier, P. Kannouche, M.P. Reck, A.R. Lehmann, R.P. Fuchs, A. Cordonnier, Co-localization in replication foci and interaction of human Y-family members, DNA polymerase pol eta and REVl protein, DNA Repair (Amst.) 3 (2004) 1503-1514.

[35] J.P. Volpe, J.E. Cleaver, Xeroderma pigmentosum variant cells are resistant to immortalization, Mutat. Res. 337 (1995) 111-117.

[36] K. Watanabe, S. Tateishi, M. Kawasuji, T. Tsurimoto, H. Inoue, M. Yamaizumi, Rad18 guides poleta to replication stalling sites through physical interaction and PCNA monoubiquitination, EMBO J. 23 (2004) 3886-3896.

[37] W. Yang, R. Woodgate, What a difference a decade makes: insights into translesion DNA synthesis, Proc. Natl. Acad. Sci. U.S.A. 104 (2007) 15591-15598. 